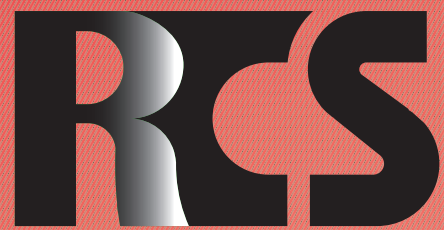

Depósito legal ppi $201502 Z U 4662$

Esta publicación científica en formato digital es continuidad de la revista impresa Depósito Legal: pp $197402 Z$ Z789

- ISSN: 1315-9518 • ISSN-E: 2477-9431

Revista de Ciencias Sociales

Universidad del Zulia. Revista de la Facultad de Ciencias Económicas y Sociales

Vol. XXVII,

No. Especial 3, 2021

Esta publicación científica en formato digital es continuidad de la revista impresa Depósito Legal: pp $197402 Z$ Z789

ISSN: $1315-9518$ 


\title{
Sistema de producción de la almendra y del cacao: Una caracterización necesaria*
}

\author{
Vera Chang, Jaime ${ }^{* *}$ \\ Álvarez Escaleras, Malena ${ }^{* * * *}$ \\ Ibáñez Astaburuaga, Alejandro ${ }^{* * * *}$
}

\section{Resumen}

El Theobroma cacao, es una especie que aporta interesante conservación de la biodiversidad, permitiendo viabilizar el cultivo como sistema biológico donde se hospedan aves, reptiles, insectos. El objetivo de este estudio consiste en analizar el sistema de producción de la almendra y del cacao, en función de generar un estándar de calidad del cacao, mediante la aplicación de características sensoriales, llevando un control adecuado de las almendras, que representan la principal materia prima para la elaboración de éste. La investigación se ejecutó en un sistema sin sombra establecido en la Finca Experimental "La Represa", ubicada en Fayta, vía Quevedo-San Carlos, provincia de Los Ríos-Ecuador, durante 114 días. Se caracterizó en un sistema de materiales mejorados, estudiando la almendra de cacao de 41 materiales hibridados; organizado en 40 tratamientos, más un testigo JHVH-10; y por unidad experimental 20 frutos fisiológicamente maduros, aplicando el análisis de componentes principales. Como resultado se obtuvo, en el análisis químico, humedad, y minerales como fósforo, cobre, hierro y zinc; grasa y energía; proteína y carbohidratos; así como potasio, calcio y magnesio. En la evaluación sensorial, sabor cacao; frutal y caramelo. Se concluye que, es importante fomentar el desarrollo sustentable del cacao en esta zona de Ecuador.

Palabras clave: Cacao; fermentación; evaluación sensorial; minerales; desarrollo sostenible.

Este documento fue auspiciado por el organizado por el Vicerrectorado Académico y la Dirección de Investigación, Innovación y Transferencia de Tecnología de la Universidad Técnica Estatal de Quevedo, Ecuador.

** Doctorante en la Universidad Americana de Europa - México, Cancún. Magíster en Procesamiento de Alimentos. Ingeniero Agropecuario. Docente de la Universidad Técnica Estatal de Quevedo, Ecuador. E-mail: jverac@uteq.edu.ec (iD) ORCID: https://orcid.org/0000-0001-6127-2307

*** Ingeniera Agropecuaria. Docente en la Facultad de Ciencias Pecuarias de la Universidad Técnica Estatal de Quevedo, Ecuador. E-mail: malena.alvarez2013@uteq.edu.ec

**** Doctor en Territorio, Urbanismo, Medio Ambiente. Ingeniero en Caminos y Proyectos. Docente de la Universidad Americana de Europa- México, Cancún. E-mail: alejandro.ibanez@aulagrupo.es

(iD) ORCID: https://orcid.org/0000-0002-8316-4005

Recibido: 2021-02-29 · Aceptado: 2021-05-18 


\title{
Almond and cocoa production system: A necessary characterization
}

\begin{abstract}
Theobroma cacao is a species that provides interesting conservation of biodiversity, allowing cultivation as a biological system where birds, reptiles and insects are housed. The objective of this study is to analyze the almond and cocoa production system, in order to generate a cocoa quality standard, through the application of sensory characteristics, carrying out adequate control of the almonds, which represent the main matter premium for the elaboration of this one. The research was carried out in a system without shade established in the Experimental Farm "La Represa", located in Fayta, via Quevedo-San Carlos, Los Ríos Province-Ecuador, for 114 days. It was characterized in a system of improved materials, studying the cocoa kernel of 41 hybridized materials; organized in 40 treatments, plus a JHVH-10 witness; and 20 physiologically mature fruits per experimental unit, applying the principal component analysis. As a result, in the chemical analysis, moisture, and minerals such as phosphorus, copper, iron and zinc were obtained; fat and energy; protein and carbohydrates; as well as potassium, calcium and magnesium. In the sensory evaluation, cocoa flavor; fruity and caramel. It is concluded that it is important to promote the sustainable development of cocoa in this area of Ecuador
\end{abstract}

Keywords: Cocoa; fermentation; sensory evaluation; minerals; sustainable development.

\section{Introducción}

El cacao (Theobroma cacao L.), es originario de América del Sur, del área del alto Amazonas, comprendiendo países como Colombia, Ecuador, Perú, y Brasil. A partir del lugar de origen, las especies se fueron difundiendo y evolucionando en dos grupos de cacao, que corresponden al cacao Criollo y Forastero (Batista, 2009). En el siglo XVIII, aparece una tercera variedad creada a partir del Criollo y el Forastero, al cual se la llama Trinitario (Páez y Espinoza, 2015).

Con la evidente tendencia del cambio climático se coloca en riesgo la diversidad, por lo cual, muchas investigaciones están enfocadas en implementar sistemas de producción incluyendo métodos agroforestales orgánicos, que mejoren la calidad de vida tanto de las personas como de aves y demás especies como reptiles, insectos, entre otros (Naoki, Gómez y Schneider, 2017; Cevallos, Urdaneta y Jaimes, 2019); entendiendo el ambiente "como un todo donde se imbrican los recursos que permiten la vida" (Finol, Hernández y
Ocando, 2019, p.205).

Por otra parte, se busca calidad ambiental para fomentar un desarrollo sustentable asociado con cacao nacional, que permita "el equilibrio entre el crecimiento económico, justicia social y respeto a la naturaleza" (Campo-Ternera, et al., 2018, p.27), puesto que aproximadamente el $71,4 \%$ de los productores desconocen el Sistema Agroforestal (SAF) (Mata, Rivero y Segovia, 2018).

El implemento de un buen sistema de clasificación, cumple la función de generar un estándar de la calidad del cacao fino de aroma, durante las etapas como la cosecha, poscosecha y comercialización, es decir, se prioriza la calidad en toda la cadena; de este sistema se sustenta el origen, aseguramiento de la calidad, fortalecimiento de productores, asistencia técnica y capacitaciones, desarrollo de estrategias y desarrollo sostenible. Otros aspectos del sistema, lo representan las características del grano, tipo de sistema fermentativo, remociones, prueba de corte, tiempo de secado, almacenamiento, costo, 
entre otros (Álvarez, et al., 2010).

En ese sentido, las propiedades sensoriales de cacao, asociadas a la calidad del chocolate, comienzan con el árbol, continúa en la cosecha, el beneficio y las distintas fases de procesamiento involucradas en el desarrollo del mismo; dando a entender que no solo la textura, el sabor, el aroma característico, o el color atractivo, hacen del chocolate el producto de cacao más popular (González, Pérez y Palomino, 2012).

Durante la fase de fermentación y secado de granos, se desarrollan precursores químicos que mediante la torrefacción se transforman en el sabor y aroma típico del cacao (Pérez, 2009); mientras que la acidez, amargor y astringencia, son un factor sensorial de notoria influencia para la pasta de cacao.

La fermentación, es afectada por el clon o variedad, el tiempo de cosecha, el método para la fermentación, grado de madurez al momento de la cosecha, entre otros (Portillo, Graziani y Cros, 2006), siendo la madurez uno de los principales problemas para el productor; aunque en el cacao nacional, la coloración va desde el verde al amarillo, en los trinitarios, la coloración es en estado verde, de color rojo oscuro, y en fase maduro, rojo claro; que es más difícil identificarlos.

Es decir, para obtener una óptima fermentación las mazorcas deben estar en una etapa de madurez, que no va a estar ni tierna ni inmadura, ni muy madura o sobre madura. Puesto que, si la baya es inmadura, no ha desarrollado totalmente los jugos del hilio o lo que se conoce como baba de cacao (mucílago). Las almendras que caen junto con otras bien desarrolladas, presentan una resistencia natural a la fermentación, afectando el sabor del chocolate, y la calidad sensorial (Quiroz, 2012).

Es así, que nació la importancia de implementar un sistema poscosecha en 41 cruces interclonales, desarrollados por la Dirección de Investigación Científica y Tecnológica perteneciente a la Universidad Técnica Estatal de Quevedo, ubicadas en la Represa, mediante la aplicación de características sensoriales, llevando un manejo postcosecha, así como control adecuado de las almendras, las cuales son las principal materia prima para la elaboración de pasta de cacao; es decir, material imprescindible para la industria nacional e internacional; por lo cual mediante la presente investigación se generó nueva información científica sobre las características sensoriales de estos híbridos, fomentando el desarrollo sostenible en la zona central de la costa ecuatoriana.

\section{Metodología}

Esta investigación es descriptiva y de campo; se ejecutó en la Finca Experimental "La Represa", propiedad de la Universidad Técnica Estatal de Quevedo (UTEQ), ubicada en el km 7.5; recinto Fayta de la vía Quevedo - San Carlos, provincia de Los Ríos-Ecuador. Su ubicación geográfica, es de $1^{\circ} 03^{\prime} 41^{\prime \prime}$ de latitud Sur y de $79^{\circ} 25^{\prime} 15^{\prime \prime}$ de longitud Oeste, a una altura de $90 \mathrm{msnm}$.

Al respecto, las variables químicas y sensoriales de la pasta de cacao estuvieron compuestas por 40 tratamientos (cruces interclonales pertenecientes al programa de cacao de la Unidad de Investigación Científica y Tecnológica de la UTEQ, más un testigo JHVH-10), con tres repeticiones y cada unidad experimental estuvo compuesta por 20 frutos fisiológicamente maduros. A continuación, se muestran los tratamientos en estudio en la Tabla 1. 
Tabla 1

Codificación de 41 cruces interclonales en estudio

\begin{tabular}{rccc}
\hline No. & Código & No. & Código \\
\hline 1 & DICYT-H -272 & 22 & DICYT-H -293 \\
2 & DICYT-H -273 & 23 & DICYT-H -294 \\
3 & DICYT-H -274 & 24 & DICYT-H -295 \\
4 & DICYT-H -275 & 25 & DICYT-H -296 \\
5 & DICYT-H -276 & 26 & DICYT-H -297 \\
6 & DICYT-H -277 & 27 & DICYT-H -298 \\
7 & DICYT-H -278 & 28 & DICYT-H -299 \\
8 & DICYT-H -279 & 29 & DICYT-H- 300 \\
9 & DICYT-H -280 & 30 & DICYT-H -301 \\
10 & DICYT-H -281 & 31 & DICYT-H -302 \\
11 & DICYT-H -282 & 32 & DICYT-H -303 \\
12 & DICYT-H -283 & 33 & DICYT-H -304 \\
13 & DICYT-H 284 & 34 & DICYT-H -305 \\
14 & DICYT-H -285 & 35 & DICYT-H -306 \\
15 & DICYT-H -286 & 36 & DICYT-H -307 \\
16 & DICYT-H -287 & 37 & DICYT-H -308 \\
17 & DICYT-H -288 & 38 & DICYT-H -309 \\
18 & DICYT-H -289 & 39 & DICYT-H -310 \\
19 & DICYT-H -290 & 40 & DICYT-H -311 \\
20 & DICYT-H -291 & 41 & TESTIGO JHVH-10 \\
21 & DICYT-H -292 & & \\
\hline
\end{tabular}

Fuente: Elaboración propia, 2020.

\subsection{Análisis de Componentes Principales (ACP)}

En lo que respecta a las variables químicas, bromatológicas y sensoriales, se empleó el APC en forma gráfica de dispersión (biplot), a las variables cuantitativas agrupadas de acuerdo a sus componentes químicos, bromatológicos y sensoriales, de acuerdo con la siguiente ecuación:

$$
r_{i j}=\frac{\operatorname{cov}\left(F_{i}, F_{j}\right)}{\sqrt{\operatorname{var}\left(F_{i}\right) \operatorname{var}\left(F_{j}\right)}}
$$

\subsection{Sistema de poscosecha del cacao}

Un sistema poscosecha, tiene un origen desde la genética del material pasando a la cosecha en campo, hasta que llega a la mesa del consumidor, en esta trazabilidad se distribuye un aporte económico en cada etapa, ante lo cual se expresa la idea de minimizar cualquier pérdida, permitiendo aumentar los ingresos del productor. Es decir, inicia en la precosecha que corresponde a la fase primaria de la producción del cacao; la segunda etapa, es la poscosecha que inicia en la cosecha del cacao hasta el consumo del producto ya elaborado (Bello, 2000; Dafermos y Vivero, 2015). 
La aplicación de la fermentación, tiene como finalidad, el fomentar el desarrollo de los precursores del sabor a chocolate, los cuales son: El amino ácido libre, péptido, y azúcares reductores. Y como resultado de esta reacción, se genera una diversidad de efectos, pigmentos marrones (melanoidinas), que ayudan con la actividad antioxidante, sabor y color del grano. La fermentación, es la remoción de la pulpa que encierra a los granos, y que ayuda al desarrollo de los precursores químicos que caracteriza al chocolate. Las fermentaciones de los granos de cacao, incluyen un sin número de especies microbianas entre estas se encuentran: Las levaduras, bacterias ácido acéticas, bacterias ácido lácticas, algunos bacillos, entre otros (Agrocalidad, 2012).

\subsection{Componentes del sistema de poscosecha del cacao}

Entre los componentes del sistema de poscosecha del cacao se encuentran: La precosecha, que representa la etapa primaria de la proiduccion del cacao, así como la poscosecha, dentro de la cual se encuentra la cosecha del cacao, la fermentación, secado y tostado del mismo.

a. Precosecha: Consiste en actividades de manejo en campo, entre las cuales se enfocan principalmente: El riego, es dar los requerimientos hídricos de la planta para su normal desarrollo; la poda, es una actividad que consiste en dar forma a la estructura de la panta hasta el control de enfermedades y plagas, las cuales pueden mermar la producción sobre todo cuando inicia; fertilización, es una actividad que consiste en suplir los requerimientos nutricionales de la planta, para optimizar la producción (Morales, et al., 2010; Sandoval, 2013; Vassallo, 2015).

b. Poscosecha: En ella se encuentra: La cosecha, en la cual el momento de la misma está determinado por el estado o el grado de madurez. En el caso del cacao, es conveniente distinguir la madurez comercial de una fisiológica; de las mazorcas, si ha cumplido su desarrollo de 150 días, porque todo eso incide sobre las operaciones sucesivas, particularmente, sobre el almacenamiento y la conservación (Enríquez, 2010; Agrocalidad, 2012).

De igual forma se halla la fermentación: En este estudio, la fermentación duró 5 días o 120 horas, durante los cuales hubo muchos cambios a nivel físico-químico dentro y fuera de los granos de cacao. El método Rohan, fue aplicado para fermentar la masa fresca del cacao (Restrepo y Burbano, 2005; Amorim, et al., 2017; Morales, et al., 2016).

También se encuentra el secado: en cuanto al secado, este se realizó de forma natural hasta que las almendras tuvieran $7 \%$ de humedad. Este es el complemento del proceso de fermentación, el cual se realizó a granos de cacao con el fin fundamental de reducir el porcentaje de humedad, para asegurar buenas condiciones al momento de ser almacenado, evitando el crecimiento de hongos y el ataque por insectos (Fito, et al., 2016).

Finalmente, el tostado y conchado: El tostado, consistió en escoger semillas de cacao, las cuales se colocaron a temperaturas entre 110 y $220^{\circ} \mathrm{C}$. en una estufa marca Mermet, hasta que el tostado fue homogenizado. Esta etapa, permitió determinar el desarrollo del aroma del cacao. Luego, se envasaron en fundas de papel debidamente codificadas en un lugar seco hasta su molienda y el refinado en pasta de cacao (Jachero, 2018). El conchado, se inició con el tostado de las almendras y consistió en una molienda de los granos, en un conchado por 72 horas hasta obtener una pasta brillosa, luego, fue puesta en moldes hasta su análisis sensorial (Espín, 2010).

\subsection{Evaluaciones sensoriales}

La evaluación de sabores básicos (cacao, acidez, amargor, astringencia), se cuantificó mediante una escala del 1 al 10 (Braudeau, 2001); de la misma se evalúan los sabores específicos (frutal, floral, nuez, caramelo o melaza), punto de partida para identificar cacaos finos con matices aromáticos que acompañen al sabor a cacao. Esta se realizó en 
los establecimientos de la Universidad Técnica Estatal de Quevedo (UTEQ) extensión "Finca la María"; con 14 catadores semi entrenados.

\subsection{Análisis químicos}

Los procesos de los análisis químicos de almendras, fueron ejecutados en el laboratorio de Química, en las instalaciones de la Universidad Tecnológica Equinoccial (UTE) de Santo Domingo.

\section{Resultados y discusión}

\subsection{Parámetros químicos}

a. Humedad: De acuerdo al análisis realizado, se indica que los mejores índices lo tienen el T36 (DICYT-H-307) con 0,99\% y el T8 (DICYT-H-279) con 0,70\%; por el contrario, el T28 (DICYT-H-299) mostró $0,11 \%$ de humedad y el T3 (DICYT-H-274) obtuvo como resultado el $0,14 \%$, ubicándose como los inferiores; con un promedio general de $0,36 \%$ (ver Tabla 2). Según los valores obtenidos, se puede demostrar, en general, que cumplen con la Norma INEN 623, la cual, como requisito expone que la pasta de cacao puede llegar a un máximo de 3\% de humedad (Instituto Ecuatoriano de Normalización [INEN], 1988).

Por su parte, Quiñones (2010), expone un valor mayor, en su investigación del efecto de Cocoanox, un cacao rico en polifenoles, en ratas espontáneamente hipertensas; menciona, que el chocolate tiene un $3 \%$ de humedad; otros autores que también presentan valores mayores, son Cadena y Herrera (2008), los cuales en su evaluación del efecto de procesamiento del cacao sobre el contenido de polifenoles y su actividad antioxidante, en molienda de las semillas tostadas (Mol) para obtener el licor de cacao presentan el $1,82 \%$; además argumentan, que la humedad disminuye durante el tostado y la molienda de las semillas. b. Materia seca: Por medio del análisis, se pudo establecer que el T28 (DICYT-H-299) presentó el 99,89\%; el T29 (DICYT-H-300) un 99,89\%; y T33 (DICYT-H-304) el 99,86\%; representando los mayores porcentajes reportados; al contrario del T7 (DICYT-H-278) que mostró un 88,57\%; el T36 (DICY-H-307) el 99,01\%; y el T19 (DICYT-H-290) un 99,22\%; apreciándose un promedio general de 99,33\% (ver Tabla 2). Amores (2012), en su estudio cadmio en suelos, almendras de chocolates; implicaciones para exportación de cacao; da a conocer que las concentraciones de sólidos de cacao en su investigación variaron entre el $40 \%$ al $100 \%$, con un promedio del $69,59 \%$, siendo que para un chocolate con un contenido de materia seca total de cacao $\geq 50 \%$, existe un rango de 0,03 a $1,56 \mathrm{mg} / \mathrm{kg}$ de cadmio y un promedio de $0,38 \mathrm{mg} / \mathrm{kg}$ de cadmio.

c. Ceniza: Como resultado del análisis, se destaca al T28 (DICYT-H-299) con un $4,03 \%$ de ceniza y el T39 (DICYT-H-310) con el 4,02\%, como mejores índices; los inferiores fueron el T4 (DICYT-H-275) con un 3,15\%; T2 (DICYT-H-273) presentó el 3,20\%; reflejando un promedio global de 3,62\% (ver Tabla 2). Reportando valores similares se encuentran Rodríguez, Pérez y Guzmán (2009), en su estudio sobre las características químicas y fisicoquímicas del licor de cacao alcalinizado con: Carbonato, bicarbonato e hidróxido de sodio; reportan valores similares de $4,00 \%, 3,20 \%$ y $3,81 \%$, respectivamente; así como Perea, Ramírez y Villamizar (2011), quien, en su caracterización fisicoquímica de materiales regionales de cacao colombiano, evidenció valores equivalentes.

Por otra parte, Díaz, et al. (2018); y El Salous, Angulo-González y Solís (2019), hallaron resultados mayores a los encontrados en el presente estudio; al igual que Vázquez-Ovando, et al. (2016), quienes en su investigación de alcaloides y polifenoles del cacao, mecanismos que regulan su biosíntesis y sus implicaciones en el sabor y aroma, obtienen el mayor valor de ceniza en la variedad cv. Criollo (almendras secas de Venezuela) con el 5,24\%. Conforme a los 


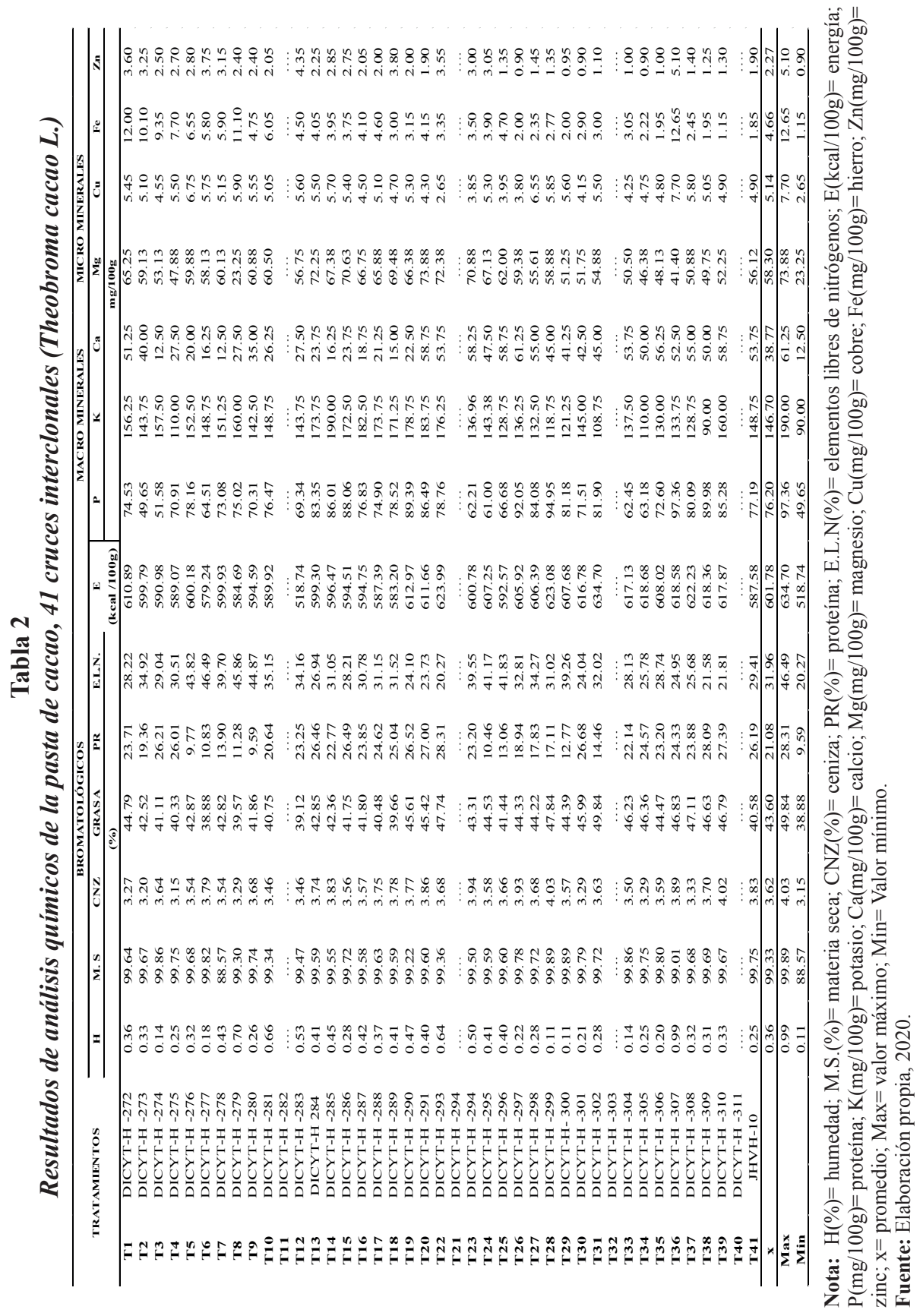


resultados expuestos, se corrobora que están dentro del rango establecido por la Norma INEN 623, en la cual se señala, un máximo de 7,5\% de ceniza (INEN, 1988).

d. Grasa: En el análisis se puede observar, que el T31 (DICYT-H-302) evidenció un 49,84\% y el T28 (DICYT-H-299) el 47,84\%, como índices mayores; al contrario del T12 (DICYT-H-283) que presentó 39,12\% y el T6 (DICYT-H-277) un $38,88 \%$; con un promedio general de 43,60\% (ver Tabla 2). Valores inferiores reportaron Rodríguez, et al. (2009), en su investigación; al igual que Sol, et al. (2016), quienes en la caracterización bromatológica de los productos derivados de cacao (Theobroma cacao L.) en la Chontalpa, Tabasco, México; reportan 46,37\%, 43,69\%; y $40,87 \%$, correspondientemente. De acuerdo a los valores reportados, se puede demostrar que el T31 (DICYT-H-302), cumple con lo establecido en la Norma INEN 623, la cual, como requisito expone que la pasta de cacao puede tener un mínimo de $48 \%$ y máximo 54\% de grasa (INEN, 1988).

Cadena y Herrera (2008), argumentan que el incremento de grasa, se presenta porque durante el proceso de molienda del cacao tostado, se facilita la liberación de la manteca contenida en las células de la semilla, permitiendo el paso a la formación de la pasta. Al respecto, Westernbrink, et al. (2009), manifiestan que todas las grasas contienen carbón, hidrogeno y oxígeno, así como algunos cacaos también contienen fósforo y nitrógeno.

e. Proteína: De acuerdo a los resultados reportados por el análisis, como índice mayor se encuentra el T22 (DICYT-H-293) con el $28,31 \%$ y el T38 (DICYT-H-309) con un 28,09\%; como inferiores se aprecian el T5 (DICYT-H-276) con 9,77\% y T9 (DICYT-H-280) con un 9,59\%; reflejando un promedio global de 21,08\% (ver Tabla 2). Difiriendo con valores inferiores, se encuentran Pérez, Álvarez y Lares (2002), los cuales, en la caracterización física y química de granos de cacao fermentado, seco y tostado de la región de Chuao, reportan 14,03\%; así como Morón, et al. (2015), quienes, en su estudio, obtención de un sustituto de chocolate tipo-pasta usando pulpa de Carao (Cassia fistula L.), presenta $8,59 \%$.

f. E.L.N (Carbohidratos): Mediante el análisis se determinó que, el T6(DICYT-H-277) mostró un 46,49\% y el T8 (DICYT-H-279) el $45,86 \%$, representando los valores superiores; mientras que, el T22 (DICYT-H-293) evidenció el 20,27\% y el T38 (DICYT-H-309) un $21,58 \%$, siendo los inferiores; con un promedio general del 31,96\% (ver Tabla 2). Al respecto, Salinas y Bolivar (2012), presentan dentro de los resultados de la composición de los carbohidratos en chocolates tipo polvo (MCG) un 86,4\%; bombón (MCA) con el $48,00 \%$ y barra leche (MCC) el 59,6\%.

Por su parte, Vázquez, et al. (2016), especifica dentro de la composición proximal, que el contenido de carbohidratos de las almendras de cacao son diferentes, de acuerdo al origen y variedad, donde cv. Criollo (almendras frescas de Sureste de México) presenta $105 \mathrm{~g} / 100 \mathrm{~g}$; cv. Criollo 0C63 (almendras secas de Venezuela) 10,39g/100g; cv. Mar 4 (almendras secas de la región de Cuyagua, Venezuela) 19,34g/100g. Ramírez y Román (2017), mencionan que tras la existencia de un gran número de carbohidratos, la estructura química determina su funcionalidad y sus características, a su vez estos influyen en el sabor, viscosidad, estructura y color, que presentan los distintos alimentos que los contienen; Mollinedo y Benavides (2014), argumenta que se los considera edulcorantes naturales y que son la principal fuente de energía para el organismo humano de fácil obtención y menor costo.

g. Energía: En base al resultado del análisis, se decreta que el T31 (DICYT-H-302) evidencia $634,70 \mathrm{kcal} / 100 \mathrm{~g}$ y el T28 (DICYT-H-299) 623,08 kcal/100g, observados como los tratamientos con los mayores valores; mientras que, el T12 (DICYT-H-283) con $518,74 \mathrm{kcal} / 100 \mathrm{~g}$ y el T6 (DICYT-H-277) con $579,24 \mathrm{kcal} / 100 \mathrm{~g}$, se muestran como los inferiores; reportándose un promedio de $601,78 \mathrm{kcal} / 100 \mathrm{~g}$ (ver Tabla 2). Afoakwa (2010), en su estudio ciencia y tecnología del chocolate, reporta un valor de $500 \mathrm{kcal} / 100 \mathrm{~g}$; el cual es inferior al expuesto. Según Morales, 
Sistema de producción de la almendra y del cacao: Una caracterización necesaria Vera Chang, Jaime; Álvarez Escaleras, Malena y Ibáñez Astaburuaga, Alejandro

García y Méndez (2012), el cacao contiene fenil-etil-amina, una sustancia química (presente en el cerebro humano) del grupo de las endorfinas cuyos efectos son conocidos, puesto que al introducirse en la sangre eleva el estado de ánimo, creando una energía altamente positiva, así como una sensación un tanto euforizante.

\subsection{Análisis de componentes principales: Variables químicas, bromatológicas}

De acuerdo al ACP, obtenido del análisis químico, y bromatológico; se observa, por un lado, una variabilidad total del $57,1 \%$, en ambos componentes para la variable E.L.N (Carbohidratos), dentro del cual se destacó el T9 (DICYT-H-280) y el T7 (DICYT-H-278), además de ser similares, al igual que el T5
(DICYT-H-276) y T25 (DICYT-H-296), presentan mayor presencia de carbohidratos. Por otro lado, para las variables Energía, Grasa y Ceniza, el T31 (DICYT-H-302) presenta mayor característica energética, T33 (DICYT-H-304) como graso, y el T37 (DICYT-H-308) propiedades de ceniza; lo cual es un indicador de la presencia de minerales, materia orgánica, micro elementos, cuya función es metabólica (Márquez, 2014).

Dentro de la variable Humedad, se destacó el T12 (DICYT-H-283), seguido por el T8 (DICYT-H-279) y T10 (DICYT-H-281), como pastas húmedas. Las variables Materia seca y Proteína, estuvieron acentuadas por el T13 (DICYT-H-284), quien presentó ambas características, una pasta proteica (ver Gráfico I). Al respecto, Bueso y Valvidia (2017) mencionan que existe una relación entre ambos, puesto que la Materia seca contiene todo, menos agua; y entre menos Materia seca, menos nutrientes.

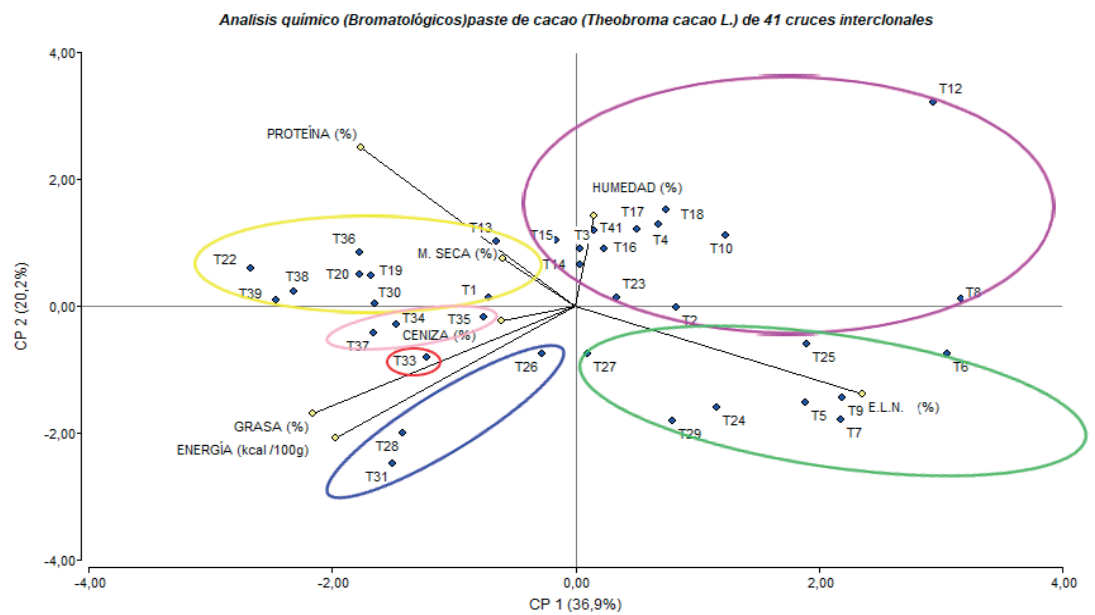

Nota: $\mathrm{H}=$ humedad; $\mathrm{MS}=$ materia seca; $\mathrm{CNZ}=$ ceniza; $\mathrm{GRA}=$ grasa; $\mathrm{PR}=$ proteína; $E . L . N .=$ elementos no nitrogenados; $\mathrm{E}=$ energía $(\mathrm{kcal} / 100 \mathrm{~g})$.

Fuente: Elaboración propia, 2020.

\section{Gráfico I: Resultado del análisis de los componentes principales bromatológicos $\%$, pasta de cacao (Theobroma cacao L.)}




\section{a. Macro minerales}

Fósforo (P): Tras el análisis, y en base a los resultados obtenidos se estableció que el T36 (DICYT-H-307) con 97,36 mg/100g y el T28 (DICYT-H-299) con 94,25 $\mathrm{mg} / 100 \mathrm{~g}$, presentan los altos valores de $\mathrm{P}$; al contrario del T3 (DICYT-H-274) con 51,58 $\mathrm{mg} / 100 \mathrm{~g}$ y el T2 (DICYT-H-273) con 49,65 mg/100g; observándose un promedio de $76,20 \mathrm{mg} / 100 \mathrm{~g}$ (ver Tabla 2). Al respecto el Instituto Colombiano de Bienestar Familiar (ICBF, 2015), reportó que dentro de las características generales del cacao (tostado y molido) realizado en sus muestras, existió un contenido de P de $500 \mathrm{mg} / 100 \mathrm{~g}$; siendo este un valor superior. Por otra parte, Afoakwa (2010) reporta un valor de $\mathrm{P}$ equivalente a 190,5 $\mathrm{mg} / 44 \mathrm{~g}$.

Potasio (K): De acuerdo a los resultados del análisis, se decreta que los mayores valores los presentan el T14 (DICYT-H-285) con $190,00 \mathrm{mg} / 100 \mathrm{~g}$ y el T20 (DICYT-H-291) con $183,75 \mathrm{mg} / 100 \mathrm{~g}$; mientras que, el T38 (DICYT-H-309) muestra 90,00 mg/100g y el T31 (DICYT-H-302) 108,75 mg/100g, los inferiores; reportándose un promedio global de 146,70 mg/100g (ver Tabla 2). En ese sentido, Escalante, Badrie y Bekele (2013) en su estudio de producción y caracterización de la calidad de la pulpa de granos de cacao de Trinidad: Efectos de niveles variables de pulpa en bebidas de cacao carbonatadas de valor agregado, expresó un contenido (K) de 6526 ppm $(6,526 \mathrm{mg} / \mathrm{g})$ en el licor de cacao.

Calcio (Ca): Mediante el análisis, se obtuvo que el T26 (DICYT-H-297) con 61,25 $\mathrm{mg} / 100 \mathrm{~g}$ y el T20 (DICYT-H-291) con 58,75 $\mathrm{mg} / 100 \mathrm{~g}$, son los mayores tratamientos con Ca; al contrario del T7 (DICYT-H-278) con $12,50 \mathrm{mg} / 100 \mathrm{~g}$ y el T18 (DICYT-H-289) con $15,00 \mathrm{mg} / 100 \mathrm{~g}$; reflejándose un promedio general de 38,77 mg/100g (ver Tabla 2). De La Cruz y Pereira (2009), en su investigación historias, saberes y sabores en torno al cacao (Theobroma cacao L.) en la subregión de Barlovento, estado Miranda, menciona el contenido medio de macro y micronutrientes en $100 \mathrm{~g}$ de chocolate; $\mathrm{Ca}$ en el chocolate puro $(63 \mathrm{mg} / 100 \mathrm{~g})$, chocolate con leche (246 $\mathrm{mg} / 100)$, chocolate blanco (306 mg/100g).

Asimismo, Pastor (2015), expone dentro de la composición y actividad farmacológica, composición química de la pulpa 6,00 $\mathrm{mg} / 100 \mathrm{~g}$ y semilla, $200,00 \mathrm{mg} / 100 \mathrm{~g}$. Por otra parte, el Instituto Colombiano de Bienestar Familiar (ICBF, 2015), reportó que dentro de las características generales del cacao (tostado y molido) realizado en sus muestras, existió un contenido de Ca de $130 \mathrm{mg} / 100 \mathrm{~g}$; el cual es un valor mayor al expuesto en la presente investigación.

Magnesio (Mg): En esta variable, de acuerdo al resultado del análisis, el T20 (DICYT-H-291) con 73,88 mg/100g y el T22 (DICYT-H-293) con 72,38 mg/100g; presentaron los mejores valores de $\mathrm{Mg}$; mientras que el T8 (DICYT-H-279) con 23,25 mg/100g y el T36 (DICYT-H-307) con $41,40 \mathrm{mg} / 100 \mathrm{~g}$, los valores inferiores; con un promedio de 58,30 mg/100g (ver Tabla 2). De Araujo, et al. (2017), en su investigación impacto de los suelos y sistemas de cultivo en la composición mineral de los granos secos de cacao; en donde la composición mineral de los clones de cacao PH-16 se sometieron a pruebas de normalidad y homoscedasticidad relacionadas con los residuos, reportan el máximo valor de $2,41 \mathrm{~g} / \mathrm{kg}(0,024 \mathrm{mg} / 100 \mathrm{~g})$ y un mínimo $1,74 \mathrm{~g} / \mathrm{kg}(0,0174 \mathrm{mg} / 100 \mathrm{~g}), \operatorname{los}$ cuales son muy inferiores.

\section{b. Micro minerales}

Cobre (Cu): De acuerdo al análisis, se determinó que los tratamientos con mayores valores fueron el T36 (DICYT-H-307) con $7,70 \mathrm{mg} / 100 \mathrm{~g}$ y el T5 (DICYT-H-276) con $6,75 \mathrm{mg} / 100 \mathrm{~g}$; al contrario del T22 (DICYT-H-293) que mostró 2,65 mg/100g y del T26 (DICYT-H-297) 3,80 mg/100g, cuyos valores fueron inferiores; con un promedio general de 5,14 mg/100g (ver Tabla 2). Lanza, et al. (2016), en su estudio evaluación del contenido de metales pesados en cacao (Theobroma cacao L.) de Santa Bárbara del Zulia, Venezuela, reportó que los niveles 
Sistema de producción de la almendra y del cacao: Una caracterización necesaria

Vera Chang, Jaime; Álvarez Escaleras, Malena y Ibáñez Astaburuaga, Alejandro

encontrados en las muestras de cacao de $\mathrm{Cu}$ se ubicaron en 21,19 y $21,36 \mathrm{mg} / \mathrm{kg}$; además, mencionan que el origen del $\mathrm{Cu}$ en las plantas de cacao se genera por el uso de fungicidas, con contenidos de óxido cuproso, en mayor o menor grado.

Hierro (Fe): El análisis, permitió establecer que el T36 (DICYT-H-) con 12,65 $\mathrm{mg} / 100 \mathrm{~g}$ y el T1 (DICYT-H-) con 12,00 $\mathrm{mg} / 100 \mathrm{~g}$, son los mayores valores registrados; mientras que el T39 (DICYT-H-) con 1,15 $\mathrm{mg} / 100 \mathrm{~g}$ y el T41 testigo (JHVH-10) con 1,85 $\mathrm{mg} / 100 \mathrm{~g}$, son los inferiores; con un promedio global de 4,66 mg/100g (ver Tabla 2). El Instituto Colombiano de Bienestar Familiar (ICBF, 2015), reportó que dentro de las características generales del cacao (tostado y molido) realizado en sus muestras, existió un contenido de $\mathrm{Fe}$ equivalente a $5,8 \mathrm{mg} / 100 \mathrm{~g}$.

Por su parte, Soto y Caballero (2011), en su estudio de adición de hierro hemo, proveniente de hemoglobina bovina a un chocolate de consumo directo, donde mesclaron $25 \mathrm{~kg}$ chocolate con HBD seleccionada a partir de una base de cálculo, sustituyendo azúcar micro pulverizada; obtuvieron que la cantidad de hierro total encontrada en la HBD fue de 245,60 mg/100g; además, mencionan que cada gramo de HBD aporta 2,45 mg de hierro total; suficiente para cubrir el $13,6 \%$ del valor diario de referencia de consumo de hierro, para personas mayores de 4 años.

Zinc (Zn): Tomando en cuenta el resultado del análisis, se determinó que los valores altos, fueron obtenidos por el T36 (DICYT-H-307) con $5,10 \mathrm{mg} / 100 \mathrm{~g}$ y el T12 (DICYT-H-283) con 4,35 mg/100g; al contrario del T29 (DICYT-H-300) que mostró $0,95 \mathrm{mg} / 100 \mathrm{~g}$ y el T30 (DICYT-H-301) 0,90 $\mathrm{mg} / 100 \mathrm{~g}$; reflejándose un promedio total de 2,27 mg/100g (ver Tabla 2). Al respecto, Quiñones (2010) reporta nutricional estándar de Cocoanox, cacao en polvo, compuestos fenólicos contenidos naturalmente en los granos de cacao con un contenido de $\mathrm{Zn}$ de $9,91 \mathrm{mg} / 100 \mathrm{~g}$.

Pérez (2014), en su investigación cacao: Salud y deporte, describe que la composición nutricional de productos típicos a partir de cacao, dependerá del producto final; cacao en polvo desgrasado $9 \mathrm{mg} / 100 \mathrm{~g}$, chocolate $1,4-2,0 \mathrm{mg} / 100 \mathrm{~g}$, chocolate con leche $0,2-$ $0,9 \mathrm{mg} / 100 \mathrm{~g}$, chocolate blanco $0,9 \mathrm{mg} / 100 \mathrm{~g}$ y soluble de cacao $2 \mathrm{mg} / 100 \mathrm{~g}$.

De igual manera, en el análisis químico, minerales, se observa una variabilidad total del 59\%, en ambos componentes. Para los micros minerales, el T36 (DICYT-H-307) comparte mayores características de $\mathrm{Cu}$ y en menores de Fe; el T6 (DICYT-H-277), se caracteriza por contener mayores proporciones de $\mathrm{Zn}$ y menores proporciones $\mathrm{Fe}$; seguido del T1 (DICYT-H-272), el cual presentó ambas propiedades en proporciones altas. Para la variable macro minerales, el T14 (DICYT-H-285), destacó por presentar mayores características de $\mathrm{K}$, seguido del T18 (DICYT-H-289). Por otra parte, el T22 (DICYT-H-288), corresponde a Mg; mientras que el T26 (DICYT-H-297) al P y Ca (ver Gráfico II).

A tenor de lo anterior, Carbajal (2013) sostiene que las concentraciones de fósforo influyen en los niveles de calcio, en caso de que esta sea baja; produciendo reducción de los niveles de calcio e incrementando la pérdida de masa ósea.

\subsection{Parámetros sensoriales}

a. Sabores básicos: Para el sabor a cacao, se identifican al T11 (DICYT-H-282), T13 (DICYT-H-284) y T23 (DICYT-H-294), con la valoración de 10; sin dejar de lado al T2 (DICYT-H-273), T4 (DICYT-H-275) y T7 (DICYT-H-278), los cuales presentan un valor de 9; mientras que, el T41 testigo (JHVH-10) con 6; por otra parte, como los inferiores el T9 (DICYT-H-280), T15 (DICYT-H-286) y T17 (DICYT-H-288) con 4; reflejándose un promedio global de 7,99 y un error de 0,39 (ver Tabla 3 ). 


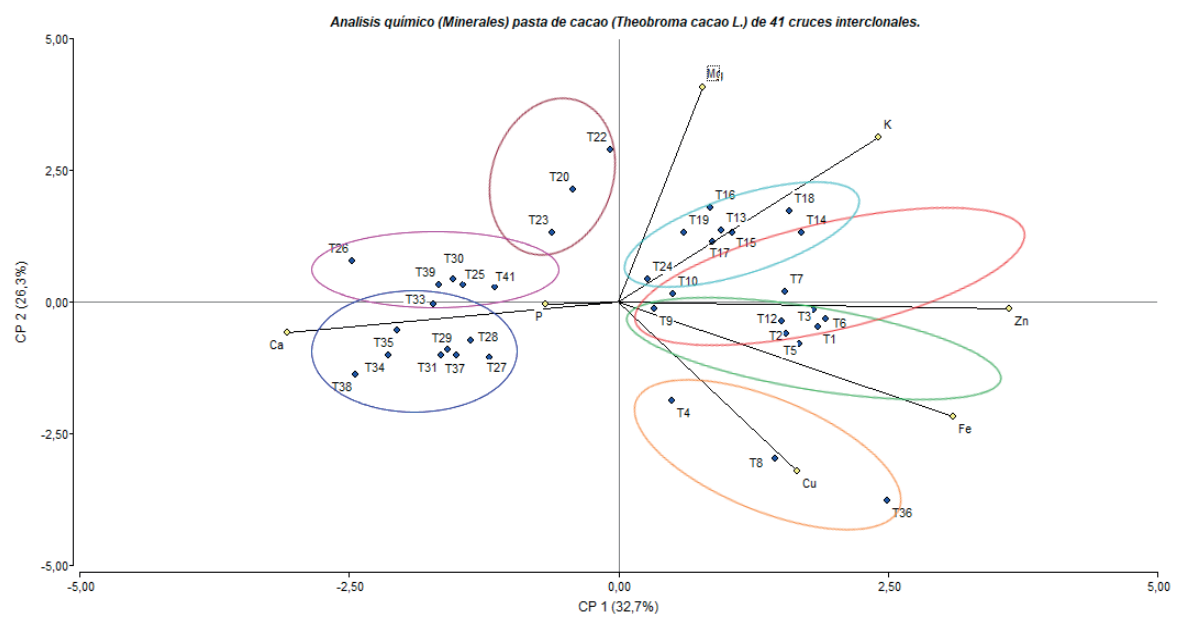

Fuente: Elaboración propia, 2020.

\section{Gráfico II: Resultado del análisis de los componentes principales minerales (macronutrientes $\mathrm{mg} / \mathbf{1 0 0 g}$; micronutrientes $\mathrm{mg} / \mathbf{1 0 0 g}$ ), pasta de cacao (Theobroma cacao L.)}

Con respecto a la acidez, el $\mathrm{T} 1$ (DICYT-H-272), T12 (DICYT-H-283), T19 (DICYT-H-290) y T23 (DICYT-H-294), se aprecian con el valor de 9; el testigo T41 (JHVH-10) con 6; el T22 (DICYT-H-293) no presenta acidez; siendo el promedio general de 7,50 y error 0,4. El T1 (DICYT-H-272), se destaca con amargor, al tener una nota de 10; asimismo, el T5 (DICYT-H-276), T7 (DICYT-H-278), T11 (DICYT-H-282), T12 (DICYT-H-283), T13 (DICYT-H-284) y T21 (DICYT-H-292), con valor 9; el testigo T41 (JHVH-10) con 6; los menos amargos, son el T19 (DICYT-H-290) con el valor de 1, así como T8 (DICYT-H-279) y T15 (DICYT-H-286) con 3; el promedio total 7,31 y error de 0,34 .

Para astringencia, se marcan el T4 (DICYT-H-275), T8 (DICYT-H-279) y T13 (DICYT-H-284) con el valor de 9; T41 (JHVH-10) con 7; el T15 (DICYT-H-286) no presenta, y el T32 (DICYT-H-303) con
1 (ver Tabla 3). Al respecto, Quintana y Gómez (2011), en su investigación de perfil sensorial del clon CCN51 T cacao producido en tres fincas del municipio de San Vicente de Chucurí; obtuvieron valores que Chimita presenta mayor intensidad en atributos sabor a cacao 3,2 y astringencia 2,9, con una acidez de 2.2 .

b. Sabores específicos: Destacándose con el sabor arriba o floral el T3 (DICYT-H-274), T4 (DICYT-H-275), T6 (DICYT-H-277), T10 (DICYT-H-281), T11 (DICYT-H-282) y T13 (DICYT-H-284) con 10; por otra parte, los que menos presentaron este sabor fueron el T15 (DICYT-H-286) con 2; el T41 (JHVH-10) con 4; mientras que, el T22 (DICYT-H-293), no calificó con la presencia del sabor.

Para frutal, con la puntuación de 10 predomina el T14 (DICYT-H-285); sin embargo, el T9 (DICYT-H-280) y T24 (DICYT-H-295), presentan una valoración 
Sistema de producción de la almendra y del cacao: Una caracterización necesaria Vera Chang, Jaime; Álvarez Escaleras, Malena y Ibáñez Astaburuaga, Alejandro

Tabla 3

Valor del perfil sensorial de la pasta de cacao (Theobroma cacao L.) de 41 híbridos interclonales

\begin{tabular}{|c|c|c|c|c|c|c|c|c|c|c|c|c|c|c|}
\hline & & \multicolumn{3}{|c|}{ Generalidades } & \multicolumn{4}{|c|}{ Sabores básicos } & \multicolumn{4}{|c|}{ Sabores específicos } & \multicolumn{2}{|c|}{ Defectos } \\
\hline Tratamiento & $\begin{array}{c}\text { Cruces } \\
\text { interclonales }\end{array}$ & 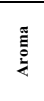 & 高 & 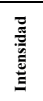 & లृ & : & 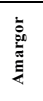 & 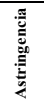 & $\frac{\bar{\pi}}{\frac{a}{x}}$ & 氖 & $\stackrel{\Xi}{\Xi}$ & 遌 & $\frac{\varrho}{\frac{1}{2}}$ & 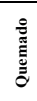 \\
\hline 1 & DICYT-H -272 & 9 & 10 & 8 & 8 & 9 & 10 & 3 & 8 & 6 & 6 & 0 & 0 & 0 \\
\hline 2 & DICYT-H -273 & 6 & 6 & 8 & 9 & 4 & 7 & 3 & 9 & 2 & 3 & 2 & 0 & 0 \\
\hline 3 & DICYT-H -274 & 9 & 8 & 8 & 7 & 4 & 8 & 6 & 10 & 0 & 0 & 0 & 5 & 0 \\
\hline 4 & DICYT-H -275 & 9 & 10 & 10 & 9 & 3 & 8 & 9 & 10 & 0 & 0 & 0 & 0 & 0 \\
\hline 5 & DICYT-H -276 & 10 & 9 & 9 & 8 & 5 & 9 & 4 & 9 & 0 & 0 & 4 & 0 & 0 \\
\hline 6 & DICYT-H -277 & 9 & 8 & 9 & 8 & 5 & 7 & 5 & 10 & 6 & 5 & 0 & 4 & 0 \\
\hline 7 & DICYT-H -278 & 9 & 10 & 9 & 9 & 2 & 9 & 2 & 9 & 2 & 0 & 4 & 3 & 0 \\
\hline 8 & DICYT-H -279 & 9 & 8 & 10 & 4 & 8 & 3 & 9 & 8 & 0 & 0 & 5 & 0 & 4 \\
\hline 9 & DICYT-H -280 & 10 & 10 & 8 & 8 & 3 & 7 & 6 & 6 & 9 & 5 & 3 & 4 & 5 \\
\hline 10 & DICYT-H -281 & 9 & 9 & 10 & 9 & 3 & 8 & 5 & 10 & 0 & 0 & 0 & 0 & 0 \\
\hline 11 & DICYT-H -282 & 10 & 9 & 9 & 10 & 6 & 9 & 3 & 10 & 8 & 0 & 0 & 2 & 0 \\
\hline 12 & DICYT-H -283 & 9 & 10 & 9 & 8 & 9 & 9 & 3 & 9 & 8 & 2 & 0 & 2 & 0 \\
\hline 13 & DICYT-H 284 & 10 & 9 & 9 & 10 & 6 & 9 & 9 & 10 & 8 & 2 & 0 & 2 & 0 \\
\hline 14 & DICYT-H -285 & 10 & 8 & 9 & 8 & 3 & 8 & 4 & 9 & 10 & 0 & 8 & 3 & 0 \\
\hline 15 & DICYT-H -286 & 8 & 6 & 5 & 4 & 2 & 3 & 0 & 2 & 1 & 1 & 0 & 0 & 0 \\
\hline 16 & DICYT-H -287 & 9 & 8 & 9 & 7 & 6 & 6 & 5 & 8 & 4 & 3 & 2 & 5 & 5 \\
\hline 17 & DICYT-H -288 & 10 & 8 & 8 & 4 & 4 & 8 & 4 & 3 & 8 & 6 & 1 & 0 & 0 \\
\hline 18 & DICYT-H -289 & 10 & 9 & 8 & 7 & 3 & 6 & 4 & 9 & 7 & 2 & 3 & 0 & 0 \\
\hline 19 & DICYT-H -290 & 8 & 6 & 4 & 7 & 9 & 1 & 8 & 5 & 3 & 1 & 8 & 7 & 2 \\
\hline 20 & DICYT-H -291 & 7 & 6 & 4 & 6 & 3 & 6 & 3 & 8 & 7 & 3 & 2 & 1 & 0 \\
\hline 21 & DICYT-H -292 & 9 & 6 & 5 & 7 & 2 & 9 & 3 & 8 & 6 & 4 & 2 & 2 & 0 \\
\hline 22 & DICYT-H -293 & 6 & 5 & 6 & 7 & 0 & 5 & 2 & 0 & 0 & 0 & 0 & 0 & 0 \\
\hline 23 & DICYT-H -294 & 10 & 9 & 8 & 10 & 9 & 8 & 7 & 3 & 8 & 2 & 4 & 2 & 1 \\
\hline 24 & DICYT-H -295 & 9 & 7 & 4 & 8 & 5 & 8 & 3 & 2 & 9 & 8 & 1 & 2 & 0 \\
\hline 25 & DICYT-H -296 & 6 & 6 & 7 & 5 & 3 & 5 & 3 & 4 & 2 & 2 & 1 & 1 & 1 \\
\hline 26 & DICYT-H -297 & 6 & 6 & 7 & 6 & 4 & 5 & 3 & 4 & 3 & 3 & 2 & 1 & 1 \\
\hline 27 & DICYT-H -298 & 6 & 6 & 7 & 7 & 5 & 5 & 6 & 6 & 4 & 1 & 1 & 0 & 1 \\
\hline 28 & DICYT-H -299 & 5 & 6 & 7 & 5 & 3 & 6 & 4 & 4 & 4 & 2 & 1 & 0 & 1 \\
\hline 29 & DICYT-H- 300 & 7 & 7 & 8 & 6 & 2 & 4 & 3 & 6 & 7 & 3 & 2 & 0 & 1 \\
\hline 30 & DICYT-H -301 & 6 & 6 & 7 & 6 & 2 & 5 & 3 & 5 & 5 & 3 & 1 & 0 & 1 \\
\hline 31 & DICYT-H -302 & 7 & 7 & 7 & 6 & 3 & 4 & 4 & 5 & 5 & 3 & 1 & 0 & 1 \\
\hline 32 & DICYT-H -303 & 9 & 10 & 6 & 5 & 2 & 7 & 1 & 3 & 6 & 2 & 2 & 0 & 0 \\
\hline 33 & DICYT-H -304 & 5 & 6 & 6 & 7 & 2 & 4 & 2 & 5 & 5 & 4 & 1 & 0 & 1 \\
\hline 34 & DICYT-H -305 & 7 & 6 & 6 & 7 & 3 & 5 & 2 & 4 & 3 & 2 & 1 & 0 & 2 \\
\hline 35 & DICYT-H -306 & 7 & 7 & 6 & 6 & 3 & 5 & 3 & 4 & 5 & 4 & 2 & 0 & 2 \\
\hline 36 & DICYT-H -307 & 7 & 7 & 7 & 7 & 4 & 5 & 2 & 4 & 5 & 3 & 1 & 1 & 2 \\
\hline 37 & DICYT-H - 308 & 8 & 7 & 6 & 7 & 3 & 6 & 3 & 4 & 4 & 3 & 1 & 1 & 2 \\
\hline 38 & DICYT-H -309 & 7 & 6 & 6 & 7 & 3 & 5 & 3 & 5 & 5 & 3 & 1 & 0 & 2 \\
\hline 39 & DICYT-H -310 & 7 & 6 & 7 & 7 & 3 & 6 & 4 & 5 & 4 & 3 & 1 & 0 & 2 \\
\hline 40 & DICYT-H -311 & ....... & $\ldots \ldots$ & ....... & ...... & $\ldots \ldots$ & ....... & ....... & $\ldots \ldots$ & $\ldots \ldots$ & $\ldots \ldots$ & ....... & ....... & $\ldots \ldots$ \\
\hline 41 & JHVH-10 & 6 & 6 & 7 & 6 & 2 & 6 & 4 & 4 & 5 & 4 & 1 & 1 & 2 \\
\hline \multicolumn{2}{|r|}{$\mathrm{x}$} & 3,87 & 3,63 & 3,43 & 3,57 & 1,89 & 3,04 & 1,87 & 2,42 & 2,63 & 1,57 & 0,9 & 0,51 & 0,59 \\
\hline \multicolumn{2}{|r|}{ Max } & 10 & 10 & 10 & 10 & 9 & 10 & 9 & 10 & 10 & 8 & 8 & 7 & 5 \\
\hline \multicolumn{2}{|r|}{ Min } & 5 & 0 & 0 & 0 & 0 & 0 & 0 & 0 & 0 & 0 & 0 & 0 & 0 \\
\hline \multicolumn{2}{|r|}{ Error } & 0,39 & 0,4 & 0,34 & 0,33 & 0,27 & 0,33 & 0,26 & 0,37 & 0,09 & 0 & 0,09 & 0,2 & 0,47 \\
\hline
\end{tabular}

Fuente: Elaboración propia, 2020.

de 9; el T41 (JHVH-10) con 5; el T3 (DICYT-H-274), T4 (DICYT-H-275), T5 (DICYT-H-276), T8 (DICYT-H-279), T10 (DICYT-H-281) y T22 (DICYT-H-293), no aplicaron a la presencia del sabor frutal; y el T15 (DICYT-H-286) con 1.
Dentro del sabor a nuez, la mayor puntuación la obtuvo el T24 (DICYT-H-295) con 8; el T41 (JHVH-10) con 4; mientras que en el T3 (DICYT-H-274), T4 (DICYT-H-275), T5 (DICYT-H-276), T7 (DICYT-H-278), T8 (DICYT-H-279), T10 (DICYT-H-281), 
T14 (DICYT-H-285) y T22 (DICYT-H-293), no existió la presencia del sabor; el T15 (DICYT-H-286), T19 (DICYT-H-290), T27 (DICYT-H-298), calificaron con 1 para nuez.

Referente a sabor caramelo, con la mayor calificación de 8, están el T14 (DICYT-H-285) y T19 (DICYT-H-292); los que no calificaron con este sabor, son el T1 (DICYT-H-272), T3 (DICYT-H-278), T4 (DICYT-H-275), T6 (DICYT-H-277), T10 (DICYT-H-281), T11 (DICYT-H-282), T12 (DICYT-H-283), T13 (DICYT-H-284), T15 (DICYT-H-286), y T22 (DICYT-H-293); en tanto que, el T24 (DICYT-H-295), T25 (DICYT-H-296), T27 (DICYT-H-298), T28 (DICYT-H-299), T30 (DICYT-H-301), T31 ((DICYT-H-302), T33 (DICYT-H-304), T34 (DICYT-H-305), T36 (DICYT-H-307), T37 (DICYT-H-308), T38 (DICYT-H-309), T39 (DICYT-H-310), T40 (DICYT-H-311), y el T41 (JHVH-10), obtuvieron la valoración de 1 (ver Tabla 3 ).

En ese sentido, Quintana, et al. (2015) en su evaluación de perfil sensorial del clon de cacao $\mathrm{T}$ cacao CCN51, expone que el licor de la región de Arauca con un 78\% de fermentación, es el que presenta un menos sabor ácido, astringente y amargo, mostrando un mayor sabor a cacao, notas de dulce, frutal y nuez; cuya calificación fue un puntaje de 7 .

c. Defectos: Perteneciente al sabor Moho, con la máxima puntuación de 7, el T19 (DICYT-H-290); con valores inferiores, T3 (DICYT-H-274) y T16 (DICYT-H-287), con 5; T6 (DICYT-H-277) y T9 (DICYT-H-280) con 4; T11 (DICYT-H-282), T12 (DICYT-H-283), T13 (DICYT-H-284), T21 (DICYT-H-292), T23 (DICYT-H-294) y T24 (DICYT-H-295), con 2; T20 (DICYT-H-291), T25 (DICYT-H-296), T26 (DICYT-H-297), T36 (DICYT-H-307), T37 (DICYT-H-308) y T41 (JHVH-10) con 1; esta presencia de moho se debe a un mal proceso de secado.

Por otra parte, para Quemado, los mayores valores lo registraron el T9 (DICYT-H-280) y T16 (DICYT-H-287), con
5; los valores inferiores, lo presentan el T8 (DICYT-H-279) con 4; T19 (DICYT-H-290), T34 (DICYT-H-305), T35 (DICYT-H-306), T36 (DICYT-H-307), T37 (DICYT-H-308), T38 (DICYT-H-309), T39 (DICYT-H-310) y T41 (JHVH-10), con 2; T23 (DICYT-H-294), T25 (DICYT-H-296), T26 (DICYT-H-297), T27 (DICYT-H-298), T28 (DICYT-H-299), T30 (DICYT-H-301) y T31 (DICYT-H-302), con 1 (ver Tabla 3); esta incidencia de quemado, se dio por falta de control de temperatura, durante el proceso de tostado.

Al respecto, Hii, et al. (2006) en su investigación de calidad de los granos de cacao secos utilizando un secador solar directo en diferentes cargas $(20,30$ y $60 \mathrm{~kg}$ ); expone que los paneles sensoriales no detectaron sabor a moho entre las muestras. El moho, ocurre cuando los frijoles no están lo suficientemente secos, están mal fermentados y almacenados en condiciones desfavorables. Aunque se observó moho en la superficie de la almendra durante el secado, especialmente en los $60 \mathrm{~kg}$ de carga, esto está restringido a la superficie externa, mientras que la punta está libre de esta contaminación.

\subsection{Análisis de componentes principales: Variables sensoriales}

En el análisis sensorial, se observa una variabilidad total del 51,4\%, en ambos componentes. El T19 (DICYT-H-289) puntúa con sabor a Caramelo; seguido del T5 (DICYT-H-276), que también presenta sabor a Astringencia; mientras que, el T4 (DICYT-H-275) califica con sabor Astringencia, solamente (ver Gráfico III); esta acción se da porque las almendras de cacao son ricas en polifenoles, el cual atribuye la sensación de amargor y astringencia de las almendras (Vázquez, et al., 2016). 
Sistema de producción de la almendra y del cacao: Una caracterización necesaria Vera Chang, Jaime; Álvarez Escaleras, Malena y Ibáñez Astaburuaga, Alejandro

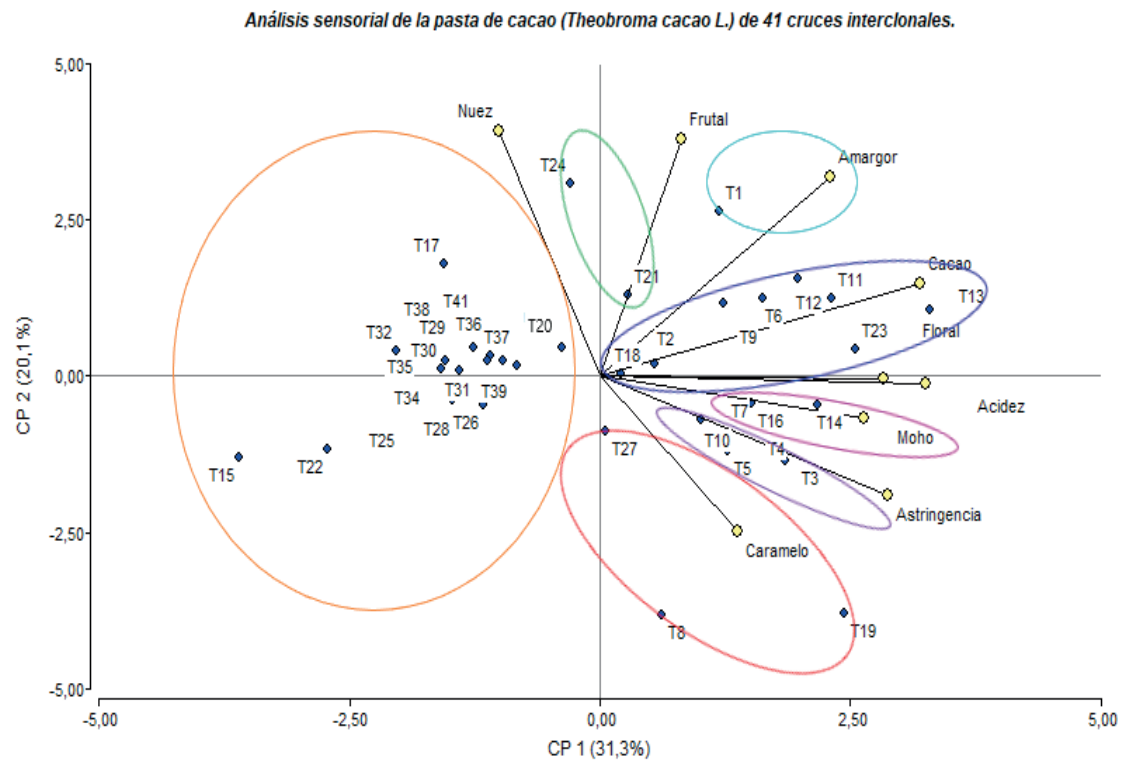

Fuente: Elaboración propia, 2020.

\section{Gráfico III: Resultado del análisis sensorial de los componentes principales, pasta de cacao (Theobroma cacao L.)}

Para sabor a Moho, el T16 (DICYT-H-287); para Floral y Acidez, T14 (DICYT-H-285) y T7 (DICYT-H-278); por otra parte, el T23 (DICYT-H-294) puntúa con sabor a Acidez y Cacao; al contrario de T13 (DICYT-H-284) el cual, califica con sabor Floral y Cacao, al igual que T11 (DICYT-H-282), T12 (DICYT-H-283), T6 (DICYT-H-277), T2 (DICYT-H-273) y T18 (DICYT-H-289). El T1 (DICYT-H-272), aplica para sabor Amargor; con sabor a Frutal, el T24 (DICYT-H-295), seguido del T21 (DICYT-H-292). El T17 (DICYT-H-288), se cataloga con sabor a Nuez, seguido del Testigo T41 (JHVH-10) (ver Gráfico III).

\section{Conclusiones}

De acuerdo, al análisis químico bromatológico, realizado en la pasta de cacao, el T36 y T8 destacaron con mejores índices de humedad; el T28 a pesar de presentar ligera fermentación, calificó como alto en porcentaje de materia seca, ceniza, grasa y como pasta que aporta energía; el T22 y T38 como pastas ricas en proteínas; así como registrando alto contenido de carbohidratos el T6 y T8.

Asimismo, en el análisis químico de minerales de la pasta de cacao, el T36 puntuó como pasta con alto contenido de fósforo, cobre, hierro, y zinc. El T20, con contenido de potasio, calcio y magnesio.

De igual manera, tras la evaluación sensorial, puntuándose con valor de 10 en sabor a cacao el T11, T13 y T23; sabor arriba el T3, T4, T6, T10, T11 y T13; con valoración de 9, el T14, T9 y T24 en sabor a frutal; por otra parte, el sabor a caramelo T14 y T19, con calificación de 8 .

Además, el cacao presenta muchos 
beneficios entre los cuales se encuentran, que ayudan al corazón reduciendo su presión sanguínea o el nivel de colesterol; posee propiedades antiinflamatorias, que regulan los triglicéridos, los fosfolípidos y el colesterol. También, contribuyen a bajar la presión arterial, ralentizan el proceso de envejecimiento y mejoran el rendimiento de los procesos mentales, incluido el de la memoria.

Finalmente, dado los hallazgos obtenidos a partir de la presente investigación, se considera importante fomentar el desarrollo sustentable del cacao en esta zona de Ecuador, implementando un buen sistema de producción, con control de calidad, por cuanto entre otros el cacao, proporciona diversos minerales como Fósforo, Potasio, Calcio, Magnesio y Zinc, necesarios en la función metabólica del ser humano; así como gran contenido de Hierro, suficiente para cubrir el valor diario de referencia de consumo del mismo, aportando energía, incrementando la producción de endorfinas cuyos efectos al introducirse en la sangre eleva el estado de ánimo de las personas.

\section{Referencias bibliográficas}

Afoakwa, E. (2010). Chocolate science and technology. Wiley-Blackwell.

Agrocalidad (2012). Guía de Buenas Prácticas Agrícolas para Cacao. Agrocalidad. https://www.agrocalidad.gob. ec/?page id $=41267$

Álvarez, C., Tovar, L., García, H., Morillo, F., Sánchez, P., Girón, C., y De Farias, A. (2010). Evaluación de la calidad comercial del grano de cacao (Theobroma cacao L.) usando dos tipos de fermentadores. Revista Cientifica UDO Agrícola, 10(1), 7687.

Amores, F. (2012). Cadmio en suelos, almendras de chocolates:
Implicaciones para exportacion de cacao. Instituto Nacional Autónomo de Investigación Agropecuaria.

Amorim, G., Reis, Q., René-Valle, R., Andrade-Sodré, G., y Moreira, S. M. (2017). Influencia de factores agroambientales sobre la calidad del clon de cacao (Theobroma cacao L.) PH-16 en la región cacaotera de Bahia, Brasil. Ecosistemas y Recursos Agropecuarios, 4(12), 579-587. https:// doi.org/10.19136/era.a4n12.1274

Batista, L. (2009). Guía Técnica: El Cultivo de Cacao. Centro para el Desarrollo Agropecuario y Forestal, Inc. (CEDAF).

Bello, J. (2000). Ciencia Bromatológica: Principios generales de los alimentos. Ediciones Díaz de Santos, S. A.

Braudeau, J. (2001). El cacao. Técnicas agricolas y producciones tropicales. Editorial Blumé.

Bueso, M., y Valvidia, G. (2017). Ficha Técnica No. 4. Nutrición ganado de engorde: Importancia de la Materia Seca. Texas Tech University. https:// www.depts.ttu.edu/icfie/Countries projects/LAC/Honduras/CAFOGAH/ fs4.pdf

Cadena, T., y Herrera, Y. M. (2008). Evaluación del efecto de procesamiento del cacao sobre el contenido de polifenoles y su actividad antioxidante (Tesis de pregrado). Universidad Insdustrial de Santander, Bucaramanga, Colombia.

Campo-Ternera, L., Amar-Sepúlveda, P., Olivero, E., y Huguett, S. (2018). Emprendimiento e innovación como motor del desarrollo sostenible: Estudio bibliométrico (2006- 2016). Revista de Ciencias Sociales (Ve), XXIV(4), 26-37.

Carbajal, Á. (2013). Manual de Nutrición y Dietética. Universidad Complutense de Madrid. 
Sistema de producción de la almendra y del cacao: Una caracterización necesaria

Vera Chang, Jaime; Álvarez Escaleras, Malena y Ibáñez Astaburuaga, Alejandro

Cevallos, M., Urdaneta, F., y Jaimes, E. (2019). Desarrollo de sistemas de producción agroecológica: Dimensiones e indicadores para su estudio. Revista de Ciencias Sociales (Ve), XXV(3), 172185.

Dafermos, G., y Vivero-Pol, J. L. (2015). Agroalimentación:

Sistema agroalimentario abierto y sustentable en Ecuador. En D. Vila-Viñas (Ed.), Buen conocer - FLOK Society (pp. 293-344). IAEN-CIESPAL.

De Araujo, Q. R., Baligar, V. C., Loureiro, G. A. H., De Souza, J., y Comerford, N. B. (2017). Impact of soils and cropping systems on mineral composition of dry cacao beans. Journal of Soil Science and Plant Nutrition, 17(2), 410-428. https://dx.doi.org/10.4067/S071895162017005000030.

De La Cruz, E., y Pereira, I. (2009). Historias, saberes y sabores en torno al cacao (Theobroma cacao L.) en la subregión de Barlovento, estado Miranda. Sapiens, 10(2), 97-120.

Díaz, L. E., Mendoza, E., Bravo, M., y Domínguez, N. (2018). Determinación de Cadmio y Plomo en almendras de cacao (Theobroma cacao), proveniente de fincas de productores orgánicos del cantón Vinces. Espirales: Revista Multidisciplinaria de Investigacion, 2(15).

El Salous, A., Angulo-González, A., y Solís, L. (2019). Acceleration of cocoa fermentation through the action of bacteria (Acetobacter aceti) and yeast (Saccharomyces cerevisiae). Espirales Revista Multidisciplinaria de Investigación, 3(28), 1-20. https:// doi.org/10.31876/er.v3i28.572

Enríquez, G. (2010). Cacao orgánico: Guía para productores ecuatorianos. Instituto Nacional de Investigaciones Agropecuarias (INIAP).
Escalante, M., Badrie, N., y Bekele, F. L. (2013). Production and quality characterization of pulp from cocoa beans from Trinidad: Effects of varying levels of pulp on valueadded carbonated cocoa beverages. Proceedings of the Caribbean Food Crops Society, 49, 238-249.

Espín, C. R. (2010). Caracterización fisicaquímica y sensorial de 13 clones de cacao (Theobroma cacao l.) tipo nacional establecidos en la finca "la represa" para obtenciòn de pasta (Tesis de pregrado). Uuniversidad Técnica Estatal de Quevedo, Quevedo, Ecuador.

Finol, W., Hernández, O., y Ocando, M. (2019). Consideraciones epistemológicas del saber ambiental. Revista de Ciencias Sociales (Ve), XXV(2), 204-216.

Fito, P., Andrés, A. M., Barat, J. M., y Albors, A. M. (2016). Introducción al secado de alimentos por aire caliente. Editorial Universitat Politécnica de Valencia.

González, Y., Pérez, E., y Palomino, C. (2012). Factores que inciden en la calidad sensorial del chocolate. Actualización en Nutrición, 13(4), 314-331.

Hii, C. L., Rahman, R. A., Jinap, S., y Che Man, Y-B. (2006). Quality of cocoa beans dried using a direct solar dryer at different loadings. Journal of the Science of Food and Agriculture, 86, 1237-1243.

Instituto Colombiano de Bienestar Familiar ICBF (2015). Tabla de composición de alimentos colombianos 2015. ICBF. https://www.icbf.gov.co/system/files/ tcac 2015 final para imprimir.pdf

Instituto Ecuatoriano de Normalización - INEN (1988). Norma técnica ecuatoriana voluntaria. INEN 623: Pasta (Masa, licor) de cacao. Requisitos. INEN. https://www.normalizacion.gob.ec/ 
buzon/normas/623.pdf

Jachero, R. D. (2018). Optimización del proceso de tostado de Theobroma Cacao L. variedad CCN-51 utilizado en la elaboración de chocolate amargo (Tesis de pregrado). Universidad de Cuenca, Cuenca, Ecuador.

Lanza, J. G., Churión, P. C., Liendo, N. J., y López, V. H. (2016). Evaluación del contenido de metales pesados en cacao (Teobroma cacao 1.) de Santa Bárbara del Zulia, Venezuela. Saber, 28(1), 106-115.

Márquez, B. M. (2014). Cenizas y grasas. Teoría del muestreo. Refrigeración y congelación de alimentos: Terminología, definiciones $y$ explicaciones (Tesis de pregrado). Universidad Nacional De San Agustin, Arequipa, Perú.

Mata, D., Rivero, M., y Segovia, E. L. (2018). Sistemas agroforestales con cultivo de cacao fino de aroma: entorno socioeconómico y productivo. Revista Cubana de Ciencias Forestales, 6(1), 103-115.

Mollinedo, M., y Benavides, G. (2014). Carbohidratos. Revista de Actualización Clínica Médica, 41, 2133-2136.

Morales, J. D. J., García, A., y Méndez, E. (2012). ¿Qué sabe usted acerca de... Cacao? Revista Mexicana de Ciencias Farmacéuticas, 43(4), 79-81.

Morales, D., Rodríguez, P., Dell' Amico, J. M., Torrecillas, A., Sánchez-Blanco, M. D. J. (2010). Efecto del estrés por nacl en el crecimiento y las relaciones hídricas en plantas de tomate (Solanum lycopersicum 1.) durante el período vegetativo. Cultivos Tropicales, 31(4), 76-81.

Morales, W., Vallejo, C., Sinche, P. D., Torres, Y., Vera, J., y Anzules, E. D. (2016). Mejoramiento de las características físico-químicas y sensoriales del cacao CCN51 a través de la adición de una enzima y levadura durante el proceso de fermentación. Revista Amazónica Ciencia y Tecnología, 5(2), 169-181.

Morón, L., Caro, Y., Gonzálezo, R. E., y Torres, É. P. (2015). Obtención de un sustituto de chocolate tipo-pasta usando pulpa de Carao (Cassia fístula L.). Información tecnológica, 26(6), 39-44. http://dx.doi.org/10.4067/ $\underline{\text { S0718-07642015000600006 }}$

Naoki, K., Gómez, M. I., y Schneider, M. (2017). Selección de diferentes sistemas de producción de cacao (Theobroma cacao, Malvaceae) por aves en Alto Beni, Bolivia - una prueba de cafetería en el campo. Ecología en Bolivia, 52(2), 100-115.

Páez, L., y Espinosa, F. (2015). Ecuador tierra del cacao. Trama.

Pastor, S. H. (2015). Columna de opinión. Biopat Perú, 1(6), 13.

Perea, J. A., Ramírez, O. L., y Villamizar, A. R. (2011). Caracterización fisicoquímica de materiales regionales de cacao colombiano. Biotecnología en el Sector Agropecuario y Agroindustrial, 9(1), 35-42.

Pérez, O. (2014). Cacao: Salud y deporte. EFDeportes.com: Revista Digital, 19(194). https://www.efdeportes.com/ efd194/cacao-salud-y-deporte.htm

Pérez, R. (2009). La calidad del cacao. Consorcio Camaren.

Pérez, E., Álvarez, C., y Lares, M. (2002). Caracterización física y química de granos de cacao fermentado, seco y tostado de la región de Chuao. Agronomía Tropical, 52(2), 161-172.

Portillo, E., Graziani, L., y Cros, E. (2006). Efecto de algunos factores postcosecha sobre la calidad sensorial del cacao criollo porcelana (Theobroma 
Sistema de producción de la almendra y del cacao: Una caracterización necesaria

Vera Chang, Jaime; Álvarez Escaleras, Malena y Ibáñez Astaburuaga, Alejandro

cacao L.). Revista de la Facultad de Agronomía, 23(1), 51-59.

Quintana, L. F., Gómez, S., García, A., y Martínez, N. (2015). Perfil sensorial del clon de cacao (Theobroma cacao L.) CCN51 (primera cosecha de 2015). @limentech Ciencia y Tecnología Alimentaria, 13(1), 60-65.

Quintana, L. F., y Goméz, S. (2011). Perfil del sabor del clon CCN51 del cacao (Theobroma cacao L.) producido en tres fincas del municipio de San Vicente de Chucurí. Revista Especializada en Ingeniería de Procesos en Alimentos y Biomateriales, 5, 45-58.

Quiñones, M. D. M. (2010). Efecto de Cocoanox, un cacao rico en polifenoles, en ratas espontáneamente hipertensas (Tesis doctoral). Universidad Complutense de Madrid, Madrid, España.

Quiroz, J. (2012). Influencia de la agronomía y cosecha sobre la calidad del cacao. Boletín Técnico, (147), 1-11.

Ramírez, J., y Román, A. D. (2017). Importancia de los carbohidratos en la industria alimentaria. Pädi Boletín Científico de Ciencias Básicas e Ingenierías del ICBI, 4(8). http://doi. org/10.29057/icbi.v5i8.2048

Restrepo, A. H., A., y Burbano, J. C. (2005). Disponibilidad térmica solar y su aplicación en el secado de granos. Scientia Et Technica, XI(27), 127-132.

Rodríguez, P., Pérez, E., y Guzmán, R. (2009). Características químicas y fisicoquímicas del licor de cacao alcalinizado con: Carbonato, bicarbonato e hidróxido de sodio. Alimentos Hoy, Revista de la Asociación Colombiana de Ciencia y Tecnología de Alimentos, 18(18).

Salinas, N., y Bolivar, W. (2012). Ácidos grasos en chocolates venezolanos y sus análogos. Anales Venezolanos de Nutrición, 25(1), 25-33.

Sandoval, R. D. (2013). Calcular la lámina de riego en vivero $y$ su pre factibilidad del cultivo de cacao (theobroma cacao l.) en la zona del cantón Milagro de la Provincia del Guayas (Tesis de pregrado). Universidad de Guayaquil, Guayaquil, Ecuador.

Sol, Á., Naranjo, J. A., Córdova, V., Ávalos, D. A., y Zaldívar, J. M. (2016). Caracterización bromatológica de los productos derivados de cacao (Theobroma cacao L.) en la Chontalpa, Tabasco, México. Revista Mexicana de Ciencias Agrícolas, 7(E-14), 2817-2830. http://doi.org/10.29312/ remexca.v0i14.453

Soto, A., y Caballero, L. A. (2011). Adición de hierro hemo, proveniente de hemoglobina bovina a un chocolate de consumo directo. Bistua: Revista de la Facultad de Ciencias Básicas, 9(1), 21-31.

Vassallo, M. (2015). Diferenciación y agregado de valor en la cadena ecuatoriana del cacao. IAEN.

Vázquez-Ovando, A., Ovando-Medina, I., Adriano-Anaya, L., Betancur-Ancona, D., y Salvador-Figueroa, M. (2016). Alcaloides y polifenoles del cacao, mecanismos que regulan su biosíntesis y sus implicaciones en el sabor y aroma. Archivos Latinoamericanos de Nutrición, 66(3), 239-254.

Westernbrink, S., Oseredczk, M., Castanheira, I., y Roe, M. (2009). Food composition databases: The EuroFIR approach to develop tools to assure the quality of the data compilation. Food Chemistry, 113(3), 759-767. https://doi. org/10.1016/i.foodchem.2008.05.112 